\title{
Tropical Grassmannians, cluster algebras and scattering amplitudes
}

\author{
James Drummond, Jack Foster, Ömer Gürdoğan and Chrysostomos Kalousios \\ School of Physics $\&$ Astronomy, University of Southampton, \\ Highfield, Southampton, SO17 1BJ United Kingdom \\ E-mail: j.m.drummond@soton.ac.uk, j.a.foster@soton.ac.uk, \\ o.c.gurdogan@soton.ac.uk, c.kalousios@soton.ac.uk
}

ABSTRACT: We provide a cluster-algebraic approach to the computation of the recently introduced generalised biadjoint scalar amplitudes related to Grassmannians $\operatorname{Gr}(k, n)$. A finite cluster algebra provides a natural triangulation for the tropical Grassmannian whose volume computes the scattering amplitudes. Using this method one can construct the entire colour-ordered amplitude via mutations starting from a single term.

KEYwords: Differential and Algebraic Geometry, Scattering Amplitudes

ARXIV EPRINT: 1907.01053 


\section{Contents}

1 Introduction 1

2 Amplitudes from volumes of dual associahedra 2

3 Tropical Grassmannians and amplitudes 4

4 The positive tropical Grassmannian from webs 4

5 The tropical Grassmannian and cluster algebras 11

$6 \operatorname{Tr}^{+}(3,6) \quad 13$

$\begin{array}{lll}6.1 & \text { Triangulating } \operatorname{Tr}^{+}(3,6) \text { with clusters } & 15\end{array}$

$\begin{array}{lll}7 & \operatorname{Tr}(3,7): \text { the amplitude from } E_{6} \text { clusters } & 17\end{array}$

$\begin{array}{lll}8 & \operatorname{Gr}(3,8): \text { redundant triangulations } & 18\end{array}$

9 Conclusions and outlook to $\operatorname{Gr}(4,8) \quad 20$

\section{Introduction}

Recently a very interesting connection between scattering amplitudes and tropical geometry has been uncovered [1, 2]. The connection outlined so far is for tree-level biadjoint $\phi^{3}$ amplitudes, which can be related to the series of tropical Grassmannians $\operatorname{Gr}(2, n)$ and a generalisation to higher Grassmannians $\operatorname{Gr}(k, n)$. Such amplitudes also have a formulation in terms of a set of scattering equations which generalise the usual scattering equations of $[3-5]$

Tropical Grassmannians are defined as a space of solutions to a set of tropical hypersurface conditions which derive from the defining Plücker relations of the Grassmannian. An important ingredient in the relation to the generalised biadjoint scattering amplitudes is the notion of positivity which singles out a particular region in the tropical Grassmannian. We describe here the tropical formulation of the Grassmannian spaces and how to select the positive region. We will see that this coincides with the criteria recently used in $[1,2]$ to determine the generalised $\phi^{3}$ amplitudes for $\operatorname{Gr}(3,6)$ and $\operatorname{Gr}(3,7)$.

We will also develop the link further and describe a relation of the positive tropical Grassmannians to certain cluster algebras, as developed by Fomin and Zelevinsky [6, 7]. These same cluster algebras have also arisen in the study of the singularities of loop amplitudes in planar $\mathcal{N}=4$ super Yang-Mills theory [8]. The cluster algebra picture provides extremely efficient calculational tools for determining the relevant positive solutions to the 
tropical hypersurface conditions, allowing for spaces of even quite large dimension to be simply constructed.

Once the positive region is obtained, the generalised biadjoint $\phi^{3}$ amplitudes can be constructed as its volume in a direct generalisation of the picture described in [9]. Such a volume can be obtained additively via a triangulation of the region into simplexes. One such triangulation is provided by the (dual of the) associated cluster polytope. For the $\operatorname{Gr}(2, n)$ cases these polytopes are the $A_{n-3}$ associahedra. In the $\operatorname{Gr}(3,6)$ case this corresponds to the $D_{4}$ polytope while in the $\operatorname{Gr}(3,7)$ case it is the $E_{6}$ polytope familiar from heptagon amplitude in planar $\mathcal{N}=4$ super Yang-Mills theory. For the $\operatorname{Gr}(3,8)$ case we can obtain a triangulation from the $E_{8}$ cluster polytope. This triangulation has the feature that it makes use of eight spurious vertices generated by the cluster algebra but not strictly needed to compute the volume. The above cases exhaust the list of finite Grassmannian cluster algebras.

A feature of the polytopes arising as positive tropical Grassmannians is that in general their facets are not all simplexes. This means that there is a redundancy in parametrising their volumes since they may be triangulated (or cut into simplexes) in multiple ways, each yielding a seemingly different but actually equivalent way of obtaining the volume. In physical language this means there are multiple ways of writing the amplitude which are in fact equivalent due to non-trivial identities between different contributions.

The non-simplicial nature of certain facets may also have a bearing on the analytic structure of loop amplitudes in planar $\mathcal{N}=4$ theory. In the case of $\operatorname{Gr}(3,7)$ it would be relevant for the heptagon amplitudes studied in $[10,11]$ where it should be related to the recently discovered property of cluster adjacency [11, 12] which forbids certain consecutive pairs of branch cuts in loop amplitudes and is related to the Steinmann relations. The non-simplicial facets can be thought of as a combination of simplexes, which corresponds in the cluster polytope to shrinking edges so that many clusters combine together.

We describe how the cases of $\operatorname{Gr}(3,6)$ and $\operatorname{Gr}(3,7)$ fit into the above picture and we extend it to the case of $\operatorname{Gr}(3,8)$ whose positive tropical version corresponds to the $E_{8}$ cluster algebra. Since all these cluster algebras are finite, the triangulation procedure works in exactly the same way for all of them. Nevertheless the correspondence of between the cluster algebra and the fan for each case contains intricacies of different nature with valuable lessons and we elaborate on these in sections dedicated to different Grassmannians.

Before this we review the interpretation of the biadjoint $\phi$ amplitude as the volume of the dual to a kinematic realisation of the associahedron. We then illustrate all the main principles of the tropical Grassmannian, its positive part and the connection to cluster algebras in the example of $\operatorname{Gr}(2,5)$.

\section{Amplitudes from volumes of dual associahedra}

In [9] a connection between biadjoint scalar amplitudes and volumes was made. The main idea is to introduce a kinematic realisation of the associahedron. This is done as follows. Given an ordered set of light-like momenta $p_{1}, \ldots, p_{n}$ satisfying momentum conservation 
one introduces dual coordinates,

$$
x_{i+1}-x_{i}=p_{i}
$$

with all indices treated modulo $n$. The $\frac{1}{2} n(n-3)$ square distances $\left(x_{i}-x_{j}\right)^{2}=X_{i j}$ can be related to Mandelstam invariants via

$$
X_{i j}=s_{i, i+1, \ldots, j-1}=\left(p_{i}+p_{i+1}+\ldots p_{j-1}\right)^{2} .
$$

Note that the momenta being null implies $X_{i, i+1}=0$. The two-particle Mandelstam invariants $s_{i j}=\left(p_{i}+p_{j}\right)^{2}$ can be related to the dual variables via

$$
s_{i j}=X_{i, j+1}+X_{i+1, j}-X_{i j}-X_{i+1, j+1} .
$$

To define the kinematic associahedron we take all $X_{i j}$ positive and choose $(n-3)$ coordinates, e.g. the $X_{1, i}$ for $i=3, \ldots,(n-1)$. The remaining $\frac{1}{2}(n-2)(n-3)$ independent variables need to be constrained in order to obtain a space of dimension $(n-3)$. To do this we impose $\frac{1}{2}(n-2)(n-3)$ conditions which we take to be of the form

$$
s_{i j}=-c_{i j}, \quad 2 \leq i<j \leq n, \quad i \leq j-2,
$$

for positive constants $c_{i j}$. The coordinates $X_{1, i}$ are then constrained to run only over a certain region: the kinematic associahedron.

For the $n=5$ example the conditions (2.4) become

$$
\begin{aligned}
& X_{35}=c_{35}+X_{13}-X_{14}, \\
& X_{25}=c_{25}+c_{35}-X_{14}, \\
& X_{24}=c_{24}+c_{25}-X_{13} .
\end{aligned}
$$

The coordinates $\left(X_{13}, X_{14}\right)$ then run over a region with the shape of a pentagon as shown in figure 1.

To obtain the dual of the kinematic associahedron it is helpful to embed it into projective space $\mathbb{P}^{n-3}$. We introduce the auxiliary point $Y=\left(1, X_{13}, X_{14}, \ldots, X_{1, n-1}\right)$. The boundary conditions $X_{i j}=0$ of the kinematic associahedron become $Y \cdot W_{i j}=0$ with $W_{i j}$ given by projective dual vectors determined by the conditions (2.4).

In the case $n=5$ we have $Y=\left(1, X_{13}, X_{14}\right)$ and

$$
\begin{aligned}
& W_{13}=(0,1,0), \\
& W_{14}=(0,0,1), \\
& W_{24}=\left(c_{24}+c_{25},-1,0\right), \\
& W_{25}=\left(c_{25}+c_{35}, 0,-1\right), \\
& W_{35}=\left(c_{35}, 1,-1\right) .
\end{aligned}
$$

These dual vectors define the dual to the $\operatorname{Gr}(2,5)$ kinematic associahedron. Its volume may be computed by first triangulating it, e.g. by picking the reference point $W_{*}=(1,0,0)$ 


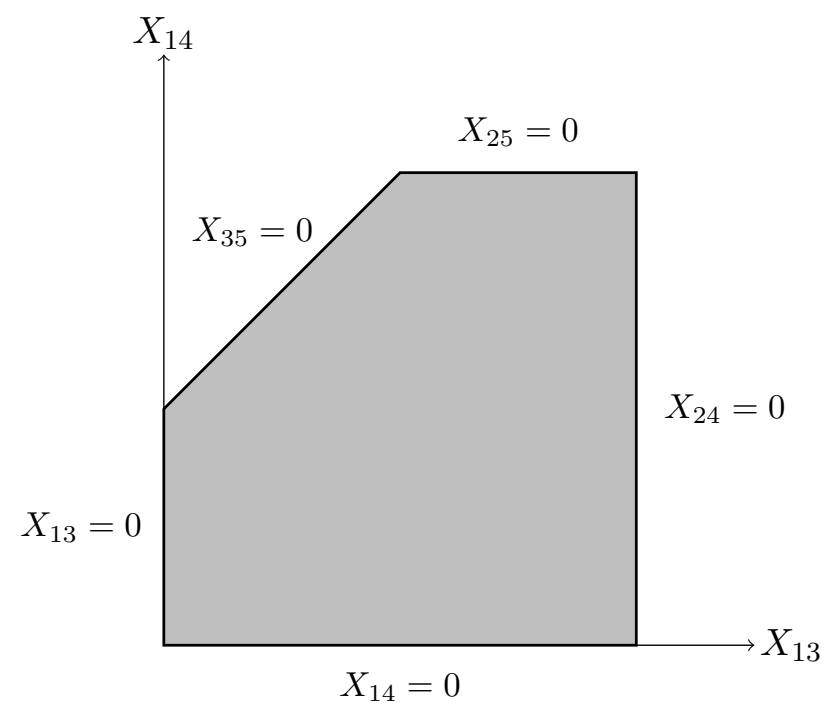

Figure 1. The shaded area is the kinematic associahedron for $n=5$.

and adding the volume of the five triangles formed by $W_{*}$ and two adjacent dual vectors according to

$$
\operatorname{Vol}\left(W_{1}, W_{2}, W_{3}\right)=\frac{\left\langle W_{1} W_{2} W_{3}\right\rangle}{\left(Y \cdot W_{1}\right)\left(Y \cdot W_{2}\right)\left(Y \cdot W_{3}\right)} .
$$

In this way we obtain the sum of five terms,

$$
\begin{aligned}
\operatorname{Vol}\left(\mathcal{A}^{*}\right) & =\frac{1}{X_{13} X_{14}}+\frac{1}{X_{14} X_{24}}+\frac{1}{X_{24} X_{25}}+\frac{1}{X_{25} X_{35}}+\frac{1}{X_{35} X_{13}}, \\
& =\frac{1}{s_{12} s_{45}}+\frac{1}{s_{45} s_{23}}+\frac{1}{s_{23} s_{15}}+\frac{1}{s_{15} s_{34}}+\frac{1}{s_{34} s_{12}}
\end{aligned}
$$

and we recognise the obtained representation as the Feynman diagram expansion for the canonically ordered biadjoint $\phi^{3}$ amplitude.

\section{Tropical Grassmannians and amplitudes}

The Grassmannian $\operatorname{Gr}(k, n)$ is the space of $k$-planes in $n$ dimensions. The Grassmannian can therefore be parametrised by a $k \times n$ complex matrix with the $k$ rows specifying a $k$ plane. Since the plane is invariant under the action of GL $(k)$ transformations one must mod out by the action of GL $(k)$, leaving a space of dimension $k(n-k)$.

The Grassmannian may also be specified in terms of the minors of the matrix. The $(k \times k)$ minors $p_{i_{1}, \ldots, i_{k}}$ (Plücker coordinates) of any matrix obey homogeneous quadratic relations (Plücker relations) obtained by antisymmetrising $(k+1)$ indices,

$$
p_{i_{1}, \ldots, i_{r},\left[i_{r+1}, \ldots i_{k}\right.} p_{\left.j_{1}, \ldots, j_{r+1}\right], j_{r+2}, \ldots, j_{k}}=0 .
$$

In the $\operatorname{Gr}(2, n)$ case the Plücker relations are given by the familiar $\left(\begin{array}{l}n \\ 4\end{array}\right)$ three-term equations

$$
p_{i j} p_{k l}-p_{i k} p_{j l}+p_{i l} p_{j k}=0, \quad 1 \leq i<j<k<l \leq n .
$$


The Plücker relations define a subspace in the Plücker space parametrised by the $\left(\begin{array}{l}n \\ k\end{array}\right)$ Plücker coordinates $p_{i_{1}, \ldots, i_{k}}$. Algebraically this space may be thought of as the ideal generated by the quadratic Plücker relations inside the ring of polynomials in the Plücker coordinates. After quotienting by a global rescaling of all Plücker coordinates the subspace satisfying the Plücker relations can be identified with the Grassmannian $\operatorname{Gr}(k, n)$ of dimension $k(n-k)$.

The original Plücker relations are actually homogeneous in $n$ independent rescalings $p_{i_{1}, \ldots, i_{k}} \rightarrow t_{i_{1}} \ldots t_{i_{k}} p_{i_{1}, \ldots, i_{k}}$ with $t_{i} \in \mathbb{C}^{*}$. If we quotient by all of these scalings instead of just the overall scaling we obtain a smaller space,

$$
\operatorname{Conf}_{n}\left(\mathbb{P}^{k-1}\right)=\operatorname{Gr}(k, n) /\left(\mathbb{C}^{*}\right)^{n-1},
$$

which has dimension $m=(k-1)(n-k-1)$ and corresponds to taking the columns of our original $(k \times n)$ to be elements of $\mathbb{P}^{k-1}$ instead of $\mathbb{C}^{k}$.

There exists a tropical version of the above construction. In tropical geometry one takes the generating relations of the ideal and replaces multiplication with addition and addition with minimum. For example the generating quadratic polynomials of the $\operatorname{Gr}(2, n)$ Plücker relations (3.2) become the tropical polynomials

$$
\min \left(w_{i j}+w_{k l}, w_{i k}+w_{j l}, w_{i l}+w_{j k}\right)
$$

which are piecewise linear maps on the space of $\left(\begin{array}{l}n \\ 2\end{array}\right)$ variables $w_{i j} \in \mathbb{R}$.

Piecewise linear maps have special surfaces between one region of linearity and another. Such surfaces are called tropical hypersurfaces and are attained when at least two of the terms of the tropical polynomial simultaneously attain the minimum. In other words the tropical polynomial (3.4) defines the following tropical hypersurfaces,

$$
\begin{aligned}
w_{i j}+w_{k l} & =w_{i k}+w_{j l} \leq w_{i l}+w_{j k} \\
\text { or } \quad w_{i j}+w_{k l} & =w_{i l}+w_{j k} \leq w_{i k}+w_{j l} \\
\text { or } \quad w_{i k}+w_{j l} & =w_{i l}+w_{j k} \leq w_{i j}+w_{k l} .
\end{aligned}
$$

When we have many polynomial relations we must simultaneously satisfy the conditions arising from each polynomial relation. In the case of $\operatorname{Gr}(2, n)$ we must simultaneously satisfy the hypersurface relations coming from every Plücker relation, i.e. for every choice of $\{i, j, k, l\}$ in $(3.2)$.

Note that for any solution $\left\{w_{i j}\right\}$, any global positive rescaling of the $w_{i j}$ will also obey the conditions. Solutions therefore form rays emanating from the origin and can be represented by an $\left(\begin{array}{l}n \\ 2\end{array}\right)$-component vector, or more generally for $\operatorname{Gr}(k, n)$ an $\left(\begin{array}{l}n \\ k\end{array}\right)$-component vector. Note also that if $\left\{w_{i j}\right\}$ are solutions of the above conditions then so are $\left\{w_{i j}+a_{i}+a_{j}\right\}$ for any set of $n$ constants $a_{i} \in \mathbb{R}$. Such a shift symmetry is referred to as lineality. In the context of generalised biadjoint scattering amplitudes it corresponds to momentum conservation.

Quotienting the space of solutions of the tropical hypersurface conditions (3.5) by a single global shift with $a_{i}=a$ corresponds to the tropical version of the Grassmannian. 
Quotienting by all shifts corresponds to the tropical version of the space $\operatorname{Conf}_{n}\left(\mathbb{P}^{k-1}\right)$. Here we are interested in the latter case where we quotient by all shifts. Despite this we will refer to the space obtained simply as the tropical Grassmannian and we use the notation $\operatorname{Tr}(k, n)$ to denote it.

The sign of the individual terms of the Plücker relations (3.2) is lost through tropicalisation. We can recover the information by identifying positive hypersurfaces as those whose defining terms in (3.2) have opposite signs [13]. This prescription defines the positive tropical Grassmannian. The positive part of $\operatorname{Tr}(2, n)$ (denoted $\operatorname{Tr}^{+}(2, n)$ ) is closely related to the dual of the kinematic associahedron that we described above and hence can be identified with the canonically ordered amplitude of the bi-adjoint $\phi^{3}$ theory. This fact is at the heart of the recent generalisation of the biadjoint amplitudes to general $\operatorname{Tr}(k, n)[1]$. In section 4 we give a more detailed introduction to the positive tropical Grassmannian following [14].

Such generalised biadjoint amplitudes can also be related to a generalisation of the scattering equations $[1,3,4]$ to $\mathbb{C P}^{k-1}$ and through them to amplitudes of a generalised scalar bi-adjoint theory [5]. Focusing for simplicity to $k=3$, we consider homogenous coordinates of $n$ particles on $\mathbb{C P}^{2}$ and form the $3 \times n$ matrix

$$
m=\left(\begin{array}{cccc}
1 & 1 & \cdots & 1 \\
x_{1} & x_{2} & \cdots & x_{n} \\
y_{1} & y_{2} & \cdots & y_{n}
\end{array}\right) .
$$

We then define the potential function

$$
S_{3}=\sum_{1 \leq i<j<k \leq n} s_{i j k} \log [i j k],
$$

where $[i j k]$ represent minors of $m$ and $s_{i j k}$ are generalized Mandelstam variables that satisfy $\sum_{j \neq k} s_{i j k}=0, \forall i$. We can now write down the amplitude of a generalised scalar theory as

$$
A_{n}^{(3)}(\alpha \mid \beta)=\frac{1}{\operatorname{vol}(\operatorname{SL}(3, \mathbb{C}))} \int \prod_{i} \mathrm{~d} x_{i} \mathrm{~d} y_{i} \delta\left(S_{3, x_{i}}\right) \delta\left(S_{3, y_{i}}\right) \operatorname{PT}(\alpha) \operatorname{PT}(\beta),
$$

where $S_{3}, i$ denotes derivative with respect to $i$ and the generalized Parke-Taylor factors involve two orderings $\alpha$ and $\beta$ and are given by

$$
\operatorname{PT}(\mathbb{I})=\frac{1}{[123][234] \cdots[n 12]} .
$$

The positive region of the tropical computation should then equal (3.8) for the canonical ordering $\alpha=\beta=\mathbb{I}$.

Let us consider explicit examples of the tropical Grassmannian [15]. The simplest case is $\operatorname{Gr}(2,4)$, defined by the single Plücker relation,

$$
p_{12} p_{34}-p_{13} p_{24}+p_{14} p_{23}=0 .
$$

In this case the tropical hypersurface conditions have three solutions (modulo lineality), given by the three possibilities in (3.5) with $\{i, j, k, l\}=\{1,2,3,4\}$. They are represented 
by the following six component vectors corresponding to the canonical ordering of the $\left\{w_{12}, w_{13}, w_{14}, w_{23}, w_{24}, w_{34}\right\}$,

$$
\begin{aligned}
& e_{12}=(1,0,0,0,0,0), \\
& e_{13}=(0,1,0,0,0,0), \\
& e_{14}=(0,0,1,0,0,0) .
\end{aligned}
$$

Of these only the first and third are positive. Note that one may not generally add solutions to obtain other solutions, the above vectors represent three distinct solutions. Note also that because of the shift symmetry $w_{i j} \mapsto w_{i j}+a_{i}+a_{j}$ the following vectors

$$
\begin{aligned}
& e_{34}=(0,0,0,0,0,1), \\
& e_{24}=(0,0,0,0,1,0), \\
& e_{23}=(0,0,0,1,0,0)
\end{aligned}
$$

are equivalent to the original three. This shift symmetry has the interpretation of momentum conservation once the solution vectors $e_{i j}$ are contracted with a canonically ordered vector of Mandelstam invariants $y=\left(s_{12}, \ldots, s_{34}\right)$ entering the massless biadjoint scattering amplitudes.

Let us now describe the $\operatorname{Gr}(2,5)$ case. In this case we have ten Plücker coordinates $p_{i j}$ and the Plücker relations are given by (3.10) and four more relations obtained from cyclic rotation of the labels. These relations give rise to the tropical hypersurface conditions (3.5) for $\{i, j, k, l\}$ given by $\{1,2,3,4\},\{1,2,3,5\},\{1,2,4,5\},\{1,3,4,5\}$ and $\{2,3,4,5\}$. Each of these five cases must be simultaneously satisfied.

We arrange the coordinates in the standard, lexicographical order,

$$
\left\{w_{12}, w_{13}, w_{14}, w_{15}, w_{23}, w_{24}, w_{25}, w_{34}, w_{35}, w_{45}\right\}
$$

and define ray vectors as

$$
\begin{aligned}
e_{12} & =(1,0,0,0,0,0,0,0,0,0), \\
e_{13} & =(0,1,0,0,0,0,0,0,0,0), \\
& \vdots \\
e_{45} & =(0,0,0,0,0,0,0,0,0,1)
\end{aligned}
$$

and so on. The vectors $e_{i j}$ so defined are simultaneously solutions to all five of the tropical hypersurface conditions.

In this case we can also combine certain solutions. For example we find that any positive linear combination $a e_{12}+b e_{34}$ with $a, b>0$ is also a solution. However no positive linear combination $a e_{12}+b e_{13}$ is a solution. We thus obtain a notion of connectivity of solutions: two solutions are connected if any positive linear combination of them is a solution. We say that there is an edge between such solutions. In the case of $\operatorname{Gr}(2,5)$ we can never combine three or more solutions to obtain another solution. In higher dimensional examples one can obtain triangles of solutions and higher dimensional faces. 


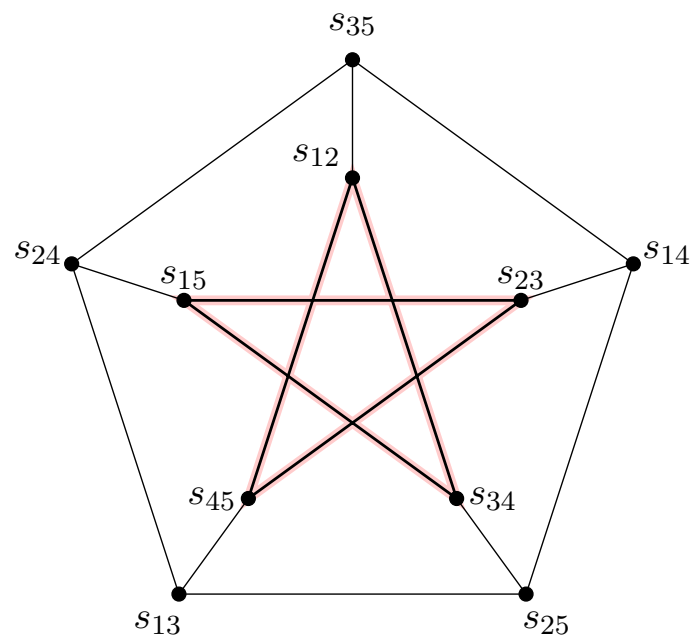

Figure 2. The 10 vertices and 15 edges of the full $\operatorname{Tr}(2,5)$ space. The highlighted star is the positive region. It corresponds to the canonical order amplitude of the scalar bi-adjoint $\phi^{3}$ theory.

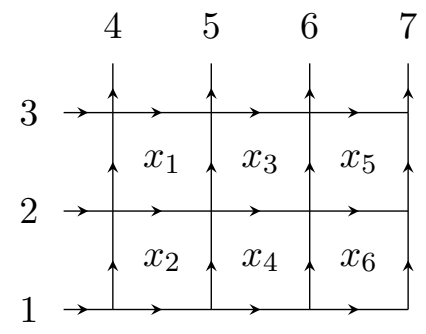

Figure 3. Example web diagram for $\operatorname{Gr}(3,7)$.

Performing permutations on the indices leads us to find 15 edges between the 10 vertices given by the $e_{i j}$. The full set of solutions corresponding to the tropical Grassmannian $\operatorname{Tr}(2,5)$ can be depicted by the Petersen graph shown in figure 2 .

The positive part $\operatorname{Tr}^{+}(2,5)$ is identified with those solutions where only the first and third possibilities in (3.5) are allowed in each of the five cases. This picks out the solutions $\left\{e_{12}, e_{23}, e_{34}, e_{45}, e_{15}\right\}$. The positive part is then given by the positive rays and the edges between them (any positive linear combination of connected positive solutions is a positive solution). The positive part is highlighted in figure 2 .

\section{The positive tropical Grassmannian from webs}

In [14] an alternative way of describing just the positive part $\operatorname{Tr}^{+}(k, n)$ was given. In this approach one introduces a grid called a web diagram with labels $\{1, \ldots, k\}$ on the horizontal edge and labels $\{(k+1), \ldots, n\}$ on the vertical edge. The squares of the grid are populated with variables $x_{i}$. In figure 3 we illustrate the general procedure in the case of $\operatorname{Tr}(3,7)$.

A Plücker coordinate is indexed by a set $K$ of $k$ distinct labels chosen from $\{1, \ldots, n\}$. We denote the set $\{1, \ldots, k\}$ by $[k]$. We may then associate a Plücker coordinate $p_{K}$ to a set of paths on the web diagram as follows. Consider sets $S$ of non-intersecting paths 

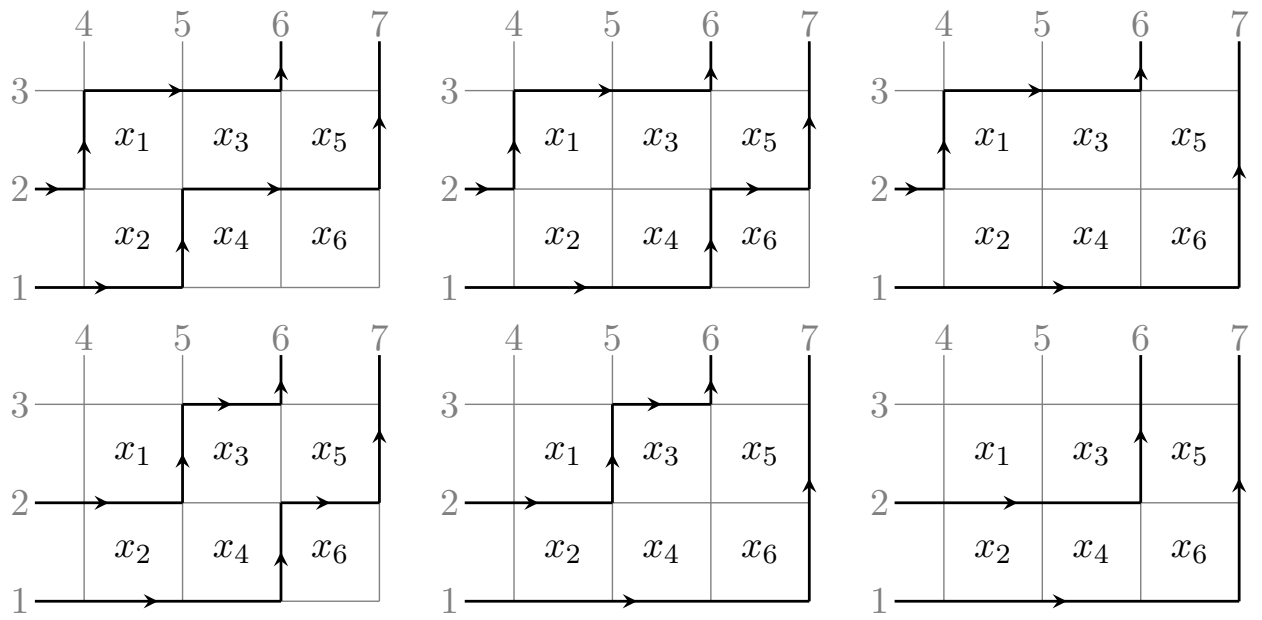

Figure 4. Possible sets of non-intersecting paths from $\{1,2\}$ to $\{6,7\}$ describing the representation (4.1) of the Plücker coordinate $p_{367}$ in $\operatorname{Gr}(3,7)$.

consistent with the arrows which go from $[k] \backslash([k] \cap K)$ to $K \backslash([k] \cap K)$. We denote the set of all such sets as $\operatorname{Path}(K)$. For each path in a given set $S$ we record the product of the variables in the squares above the path (if there are no squares above the path we record the value 1). For a set $S$ of paths we take the product over all paths in the set which we

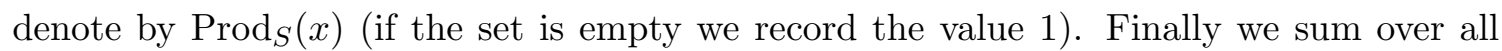
possible choices of sets $S$ of such non-intersecting paths, i.e. we sum over $S \in \operatorname{Path}(K)$,

$$
p_{K}=\sum_{S \in \operatorname{Path}(K)} \operatorname{Prod}_{S}(x) .
$$

The procedure is best illustrated with an example: consider the Plücker coordinate $p_{367}$ in the case illustrated in figure 3 . We need to consider sets of non-intersecting paths from $\{1,2\}$ to $\{6,7\}$. We find the possible choices illustrated in figure 4 . The final result for the Plücker coordinate is therefore,

$$
\begin{aligned}
p_{367}= & x_{1} x_{2} x_{3} x_{5}+x_{1} x_{2} x_{3} x_{4} x_{5}+x_{1} x_{2} x_{3} x_{4} x_{5} x_{6} \\
& +x_{1}^{2} x_{2} x_{3} x_{4} x_{5}+x_{1}^{2} x_{2} x_{3} x_{4} x_{5} x_{6}+x_{1}^{2} x_{2} x_{3}^{2} x_{4} x_{5} x_{6} .
\end{aligned}
$$

To consider the tropical Grassmannian we tropicalise the resulting polynomial, replacing multiplication with addition and addition with minimum to obtain $w_{K}$.

Following exactly the same logic for the simpler example of $\operatorname{Gr}(2,5)$ we obtain (as in [14])

$$
\begin{array}{ll}
p_{1 i}=p_{23}=1, & w_{1 i}=w_{23}=0, \\
p_{24}=1+x_{1}, & w_{24}=\min \left(0, \tilde{x}_{1}\right), \\
p_{25}=1+x_{1}+x_{1} x_{2}, & w_{25}=\min \left(0, \tilde{x}_{1}, \tilde{x}_{1}+\tilde{x}_{2}\right), \\
p_{34}=x_{1}, & w_{34}=\tilde{x}_{1}, \\
p_{35}=x_{1}+x_{1} x_{2}, & w_{35}=\min \left(\tilde{x}_{1}, \tilde{x}_{1}+\tilde{x}_{2}\right), \\
p_{45}=x_{1} x_{2}, & w_{45}=\tilde{x}_{1}+\tilde{x}_{2} .
\end{array}
$$




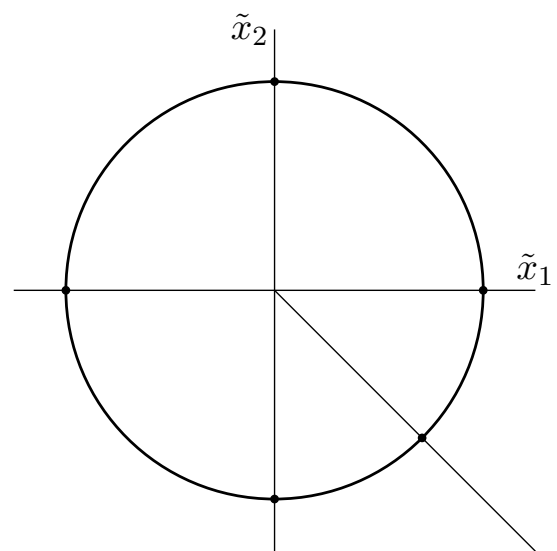

(a) $\operatorname{The}^{+}(2,5)$ polyhedral fan.

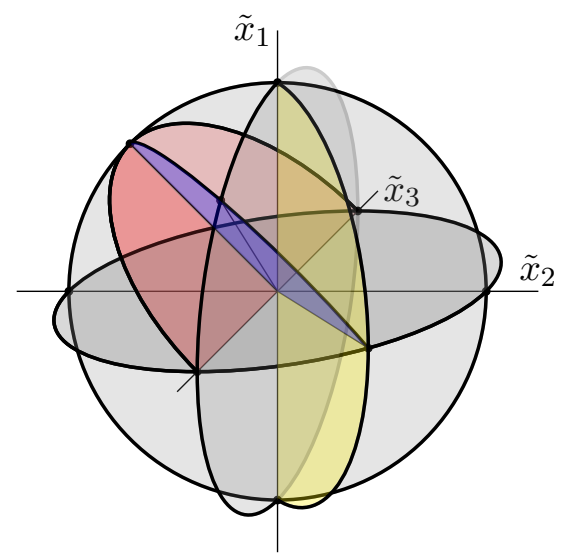

(b) $\operatorname{The}^{+}(2,6)$ polyhedral fan.

Figure 5. The intersection of the $\operatorname{Tr}^{+}(2, n)$ fan with the unit sphere $S^{n-4}$ gives the dual of the $\operatorname{Gr}(2, n)$ associahedron. Notice the $\operatorname{Tr}^{+}(2,5)$ subfan on the $\left(\tilde{x}_{1}, \tilde{x}_{2}\right)$ plane of $\operatorname{Tr}^{+}(2,6)$.

The resulting tropical minors are piecewise linear functions in the space parametrised by $\left(\tilde{x}_{1}, \tilde{x}_{2}\right)$. Each such function defines tropical hypersurfaces in exactly the same way as before. Taking the union over the tropical hypersurfaces gives rise to a fan with five domains of linearity separated by five rays as illustrated in figure 5a. We may label the five rays by

$$
\left\{\mathbf{e}_{1}, \mathbf{e}_{2},-\mathbf{e}_{1},-\mathbf{e}_{2}, \mathbf{e}_{1}-\mathbf{e}_{2}\right\}
$$

where $\mathbf{e}_{1}$ and $\mathbf{e}_{2}$ are the two-component vectors,

$$
\mathbf{e}_{1}=(1,0), \quad \mathbf{e}_{2}=(0,1) .
$$

More generally, the tropical minors in $\operatorname{Tr}^{+}(k, n)$ define a polyhedral fan in the $(k-$ 1) $(n-k-1)$-dimensional space of $\tilde{x}_{i}$ variables with many domains of linearity separated by walls of codimension one. The walls intersect in surfaces of codimension two and so on all the way down to individual rays of dimension one defined by the multiple intersection of (at least) $((k-1)(n-k-1)-1)$ walls. We illustrate the fan obtained in the case of $\operatorname{Tr}^{+}(2,6)$ in figure $5 \mathrm{~b}$.

The five rays we have obtained correspond to the five positive rays among the set (3.14). We may verify this by evaluating the tropical minors $w_{i j}$ in (4.3) on the five rays $\left\{\mathbf{e}_{1}, \mathbf{e}_{2}\right.$, $\left.-\mathbf{e}_{1},-\mathbf{e}_{2}, \mathbf{e}_{1}-\mathbf{e}_{2}\right\}$. For example if we evaluate the ten-component vector of the $w_{i j}$ on $\mathbf{e}_{1}$ we obtain the vector

$$
\mathbf{e}_{1} \mapsto \operatorname{ev}\left(\mathbf{e}_{1}\right)=(0,0,0,0,0,0,0,1,1,1) \sim(1,0,0,0,0,0,0,0,0,0)=e_{12} .
$$

where the equivalence corresponds to the lineality shift $w_{i j} \rightarrow w_{i j}+a_{i}+a_{j}$ with $\left(a_{1}, a_{2}, a_{3}\right.$, $\left.a_{4}, a_{5}\right)=\frac{1}{2}(1,1,-1,-1,-1)$. Doing the same for each of the five rays in (4.3) we indeed obtain the ten-component vectors $\left\{e_{12}, e_{45}, e_{23}, e_{15}, e_{34}\right\}$, precisely the five positive rays in the list of ten solutions given in (3.14). The regions between the rays in figure $5 \mathrm{a}$ then correspond to the edges between the positive rays in figure 2 . 
We may also recover the rays (4.3) from $\left\{e_{12}, e_{45}, e_{23}, e_{15}, e_{34}\right\}$ by tropically evaluating the coordinates $x_{1}$ and $x_{2}$ which are given by

$$
\begin{aligned}
& x_{1}=\frac{p_{12} p_{34}}{p_{14} p_{23}}, \quad \longrightarrow \quad \tilde{x}_{1}=w_{12}+w_{34}-w_{14}-w_{23}, \\
& x_{2}=\frac{p_{13} p_{45}}{p_{15} p_{34}}, \quad \longrightarrow \quad \tilde{x}_{2}=w_{13}+w_{45}-w_{15}-w_{34} .
\end{aligned}
$$

So for example the vector $e_{12}$ evaluates to $(1,0)=\mathbf{e}_{1}$ and the vector $e_{34}$ evaluates to $(1,-1)=\mathbf{e}_{1}-\mathbf{e}_{2}$.

Note that the rays (4.3) we have obtained from the tropical minors (4.3) correspond to the dual vectors (2.6) after dropping their first components. For example we have

$$
W_{24}=\left(c_{24}+c_{25},-1,0\right) \sim-\mathbf{e}_{1} .
$$

The first component of the dual vector $W_{24}$ may be recovered by demanding for example

$$
Y \cdot W_{24}=y \cdot \operatorname{ev}\left(-e_{1}\right)=s_{23}=X_{24},
$$

where we recall $Y=\left(1, X_{13}, X_{14}\right)$ and $y=\left(s_{12}, \ldots, s_{45}\right)$. Since the dual vectors are equivalent to the defining constraints of the kinematic associahedron, this gives us a way to recover the kinematic associahedron from the tropical minors.

The expressions of the web variables $x_{i}$ in terms of Plücker coordinates in fact identifies them with the cluster $\mathcal{X}$-variables of $[6,7]$ for the initial cluster of the $\operatorname{Gr}(2,5)$ cluster algebra. Indeed more generally the web variables are identified with the $\mathcal{X}$-coordinates of the initial cluster for any $\operatorname{Gr}(k, n)$. As we now outline, we can use the algebraic machinery of the cluster algebra to generate all the ray vectors describing the positive tropical Grassmannian $\operatorname{Tr}^{+}(k, n)$.

\section{The tropical Grassmannian and cluster algebras}

As mentioned above, we can identify cluster $\mathcal{X}$-coordinates with web variables. As we shall see we can also identify the ray vectors with cluster $\mathcal{A}$-coordinates. This allows us to generalise the notion of mutation to these rays such that we can generate all rays in the fan in a cluster algebraic way [16]. For a description of the relation between cluster algebras and polyhedral fans, see also [17]. Before we demonstrate this it is useful to revisit mutation for $\mathcal{A}$-coordinates in Grassmannian cluster algebras.

A $\operatorname{Gr}(k, n)$ cluster is identified by its $m=(k-1)(n-k-1)$ unfrozen nodes, $n$ frozen nodes, and an $(m+n) \times(m+n)$ exchange matrix $B$ which encodes the connectivity of the nodes within the cluster. The first $m$ rows and columns correspond to the arrows between the unfrozen nodes. Mutating an unfrozen node $k$ transforms $B$ to $B^{\prime}$ given by

$$
b_{i j}^{\prime}= \begin{cases}-b_{i j} & \text { if } i=k \text { or } j=k . \\ b_{i j}+\left[-b_{i k}\right]_{+} b_{k j}+b_{i k}\left[b_{k j}\right]_{+} & \text {otherwise }\end{cases}
$$


where $[x]_{+}=\max (x, 0)$. The mutated node also transforms, given by

$$
a_{k}^{\prime}=\frac{1}{a_{k}} \prod_{i=1}^{m+n} a_{i}^{\left[b_{i k}\right]_{+}}+\prod_{i=1}^{m+n} a_{i}^{\left[-b_{i k}\right]_{+}} .
$$

Generalising mutations to rays requires additional information, namely an additional matrix $C$ (the coefficient matrix), its mutation given by ${ }^{1}$

$$
c_{i j}^{\prime}= \begin{cases}-c_{i j} & \text { if } j=k . \\ c_{i j}-\left[c_{i k}\right]_{+} b_{k j}+c_{i k}\left[b_{k j}\right]_{+} & \text {otherwise. }\end{cases}
$$

To each unfrozen $\mathcal{A}$-coordinate we associate a ray vector $\mathbf{g}$. We start by constructing the initial cluster such that the $m$ unfrozen nodes are the $m$ basis vectors for $\mathbb{R}^{m}$ and $C$ is the identity

$$
\mathbf{g}_{l}=\mathbf{e}_{l}, \quad l=(1, \ldots, m), \quad C=\mathbb{I}_{m} .
$$

We then select a node $k$ to mutate on, following the mutation rule

$$
\begin{aligned}
\mathbf{g}_{l}^{\prime} & =\mathbf{g}_{l}, \quad \text { for } \quad l \neq k \\
\mathbf{g}_{k}^{\prime} & =-\mathbf{g}_{k}+\sum_{i=1}^{n}\left[-b_{i k}\right]_{+} \mathbf{g}_{i}+\sum_{j=1}^{n}\left[c_{j k}\right]_{+} \mathbf{b}_{j}^{0}
\end{aligned}
$$

where $\mathbf{b}_{j}^{0}, j \in\{1, \ldots, m\}$ corresponds to the $\mathrm{jth}$ column of $B^{0}$, the exchange matrix for the initial cluster. We can then repeat this process as many times as required to generate a vector for each unfrozen $\mathcal{A}$-coordinate. In the cases where the cluster algebra is of finite type (in this context the cases are $\operatorname{Gr}(2, n), \operatorname{Gr}(3,6), \operatorname{Gr}(3,7)$ and $\operatorname{Gr}(3,8)$ ) we obtain a finite cluster polytope by performing all mutations where each vertex is associated to a cluster. Each face of the polytope is associated to an unfrozen $\mathcal{A}$-coordinate $a$ and also by the above procedure a vector $\mathbf{g}$.

The advantage of having the relation of the positive tropical fan to the cluster algebra is that it gives us a very easy algebraic way to generate the relevant ray vectors to describe the fan. Once we have the fan we can embed it into the original Plücker space using the tropical minors and compute its volume to obtain the generalised scattering amplitude.

The resulting polytope in the simplest case is given in figure 6a. It has five clusters connected in the shape of a pentagon. This pentagon is the dual of the pentagon obtained from intersecting the fan illustrated in figure $5 \mathrm{a}$ with the unit circle; its edges are labelled with ray vectors (4.3).

In fact for $\operatorname{Gr}(2, n)$ the polytope obtained by intersecting the positive tropical fan with the unit sphere is always the dual polytope of the $\operatorname{Gr}(2, n)$ associahedron or Stasheff polytope. For example in figure $6 \mathrm{~b}$ we show the vectors associated to the faces of the $A_{3}$ associahedron. The dual polytope coincides with the intersection of the $\operatorname{Gr}(2,6)$ positive tropical fan with the unit sphere given in figure 5b.

For the other finite cases the tropical positive fan gives polytopes that are closely related to the duals of the cluster polytopes as we now describe.

\footnotetext{
${ }^{1}$ Note that we have modified slightly the mutation rule of the coefficient matrix of [16] so that the $\mathbf{g}$ vectors defined by (5.4), (5.5) match precisely the ray vectors for $\operatorname{Tr}^{+}(k, n)$ as defined in section 4 .
} 


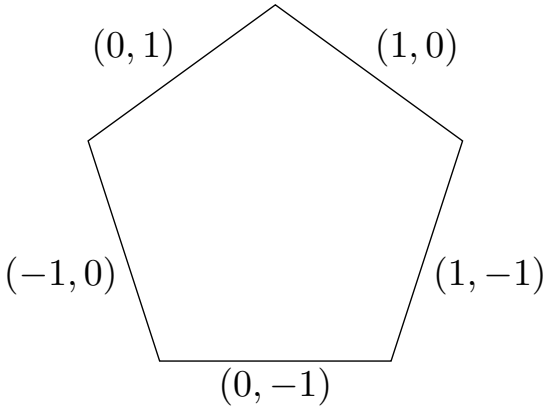

(a) $\operatorname{The}^{+}(2,5)$ polytope labelled by rays.

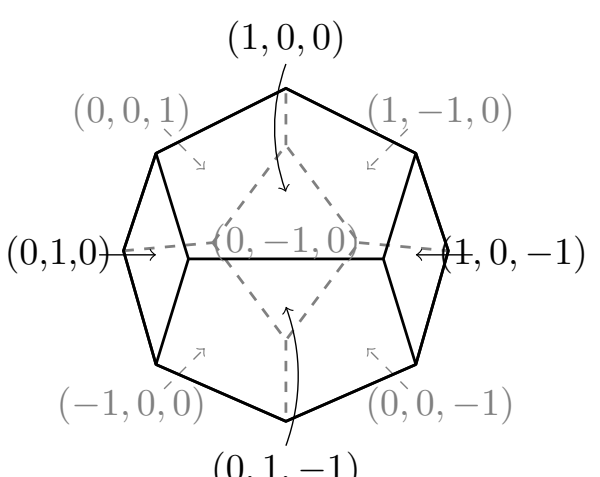

(b) $\operatorname{The}^{+} \operatorname{Tr}^{+}(2,6)$ polytope with the faces labelled by rays.

Figure 6. The cluster polytopes pictured here are the dual polyhedra of those arising from the fans shown in figure 5 .

\section{$6 \operatorname{Tr}^{+}(3,6)$}

Let us now consider the first case of the generalised biadjoint amplitudes which was addressed in [1]. In analogy to the $\operatorname{Gr}(2, n)$ cases of the previous section, the generalised amplitude for higher $k$ and $n$ can be interpreted as the volume of the computed by triangulating the relevant $\operatorname{Tr}^{+}(3,6)$ fan.

Following [15] we start by considering by the Plücker relations of $\operatorname{Gr}(3,6)$, of which there are two kinds, three-term relations and four-term relations,

$$
\begin{aligned}
p_{123} p_{145}+p_{125} p_{134}-p_{124} p_{135} & =0, \ldots \\
p_{123} p_{456}-p_{156} p_{234}+p_{146} p_{235}-p_{145} p_{236} & =0, \ldots
\end{aligned}
$$

While one can combinatorially generate many relations, only 35 of them are linearly independent.

We then tropicalise these polynomials in Plückers to obtain

$$
\begin{aligned}
& \min \left(w_{123}+w_{145}, w_{125}+w_{134}, w_{124}+w_{135}\right), \ldots \\
& \min \left(w_{123}+w_{456}, w_{156}+w_{234}, w_{146}+w_{235}, w_{145}+w_{236}\right), \ldots
\end{aligned}
$$

As before the tropical polynomials define regions of linearity in the tropical Plücker space $\mathbb{R}^{20}$ separated by hypersurfaces defined as the set of points at which the two smallest arguments of the min functions are equal. Consider for instance, the first tropical polynomial in (6.2). It gives rise to a boundary between two cones if one of the following is satisfied:

$$
\begin{array}{ll} 
& w_{123}+w_{145}=w_{125}+w_{134} \leq w_{124}+w_{135} \\
\text { or } & w_{123}+w_{145}=w_{124}+w_{135} \leq w_{125}+w_{134} \\
\text { or } & w_{124}+w_{135}=w_{125}+w_{134} \leq w_{123}+w_{145}
\end{array}
$$




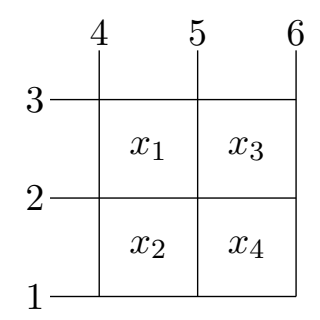

Figure 7. The web diagram for $\operatorname{Gr}(3,6)$.

This polytope contains 65 vertices [15]. As above we denote the unit vectors in the $w_{i j k}$ directions by $e_{i j k}$. These vectors give 20 of the vertices. A further 15 are of the form

$$
f_{i j k l}=e_{i j k}+e_{j i l}+e_{i k l}+e_{j k l} .
$$

The remaining 30 are of the form (for $\{i, j, k, l, m, n\}$ distinct)

$$
g_{i j, k l, m n}=f_{i j k l}+e_{k l m}+e_{k l n} .
$$

The part of the polytope that is relevant for a planar ordering is its positive part $\operatorname{Tr}^{+}(3,6)$. In [1] the positive vertices were determined by requiring compatibility with a planar ordering for the scattering amplitude. Here we identify the positive rays by requiring that they satisfy the hypersurface conditions generated by monomials in the Plücker coordinates with opposite signs as we described in section 3. This leaves us with 16 rays out of 65 , coinciding precisely with the set of [1]. They are $e_{123}$ and cyclic, $f_{1234}$ and cyclic and $g_{12,34,56}, g_{23,45,61}, g_{34,12,56}$ and $g_{45,23,61}$.

The $\mathrm{Gr}^{+}(3,6)$ web diagram shown in figure 7 produces a matrix with following piecewise linear tropical minors $[13,14]$,

$$
\begin{aligned}
& w_{12 i}=w_{134}=w_{234}=0 \\
& w_{135}=\min \left(0, \tilde{x}_{1}\right) \\
& w_{136}=\min \left(0, \tilde{x}_{1}, \tilde{x}_{1}+\tilde{x}_{3}\right) \\
& w_{145}=\tilde{x}_{1} \\
& w_{146}=\min \left(\tilde{x}_{1}, \tilde{x}_{1}+\tilde{x}_{3}\right) \\
& w_{156}=\tilde{x}_{1}+\tilde{x}_{3} \\
& w_{235}=\min \left(0, \tilde{x}_{1}, \tilde{x}_{1}+\tilde{x}_{2}\right) \\
& w_{236}=\min \left(0, \tilde{x}_{1}, \tilde{x}_{1}+\tilde{x}_{2}, \tilde{x}_{1}+\tilde{x}_{3}, \tilde{x}_{1}+\tilde{x}_{2}+\tilde{x}_{3}, \tilde{x}_{1}+\tilde{x}_{2}+\tilde{x}_{3}+\tilde{x}_{4}\right), \\
& w_{245}=\min \left(\tilde{x}_{1}, \tilde{x}_{1}+\tilde{x}_{2}\right) \\
& w_{246}=\min \left(\tilde{x}_{1}, \tilde{x}_{1}+\tilde{x}_{2}, \tilde{x}_{1}+\tilde{x}_{3}, \tilde{x}_{1}+\tilde{x}_{2}+\tilde{x}_{3}, \tilde{x}_{1}+\tilde{x}_{2}+\tilde{x}_{3}+\tilde{x}_{4}\right), \\
& w_{256}=\min \left(\tilde{x}_{1}+\tilde{x}_{3}, \tilde{x}_{1}+\tilde{x}_{2}+\tilde{x}_{3}, \tilde{x}_{1}+\tilde{x}_{2}+\tilde{x}_{3}+\tilde{x}_{4}\right), \\
& w_{345}=\tilde{x}_{1}+\tilde{x}_{2}, \\
& w_{346}=\min \left(\tilde{x}_{1}+\tilde{x}_{2}, \tilde{x}_{1}+\tilde{x}_{2}+\tilde{x}_{3}, \tilde{x}_{1}+\tilde{x}_{2}+\tilde{x}_{3}+\tilde{x}_{4}\right), \\
& w_{356}=\min \left(\tilde{x}_{1}+\tilde{x}_{2}+\tilde{x}_{3}, \tilde{x}_{1}+\tilde{x}_{2}+\tilde{x}_{3}+\tilde{x}_{4}, 2 \tilde{x}_{1}+\tilde{x}_{2}+\tilde{x}_{3}+\tilde{x}_{4}\right), \\
& w_{456}=2 \tilde{x}_{1}+\tilde{x}_{2}+\tilde{x}_{3}+\tilde{x}_{4} .
\end{aligned}
$$




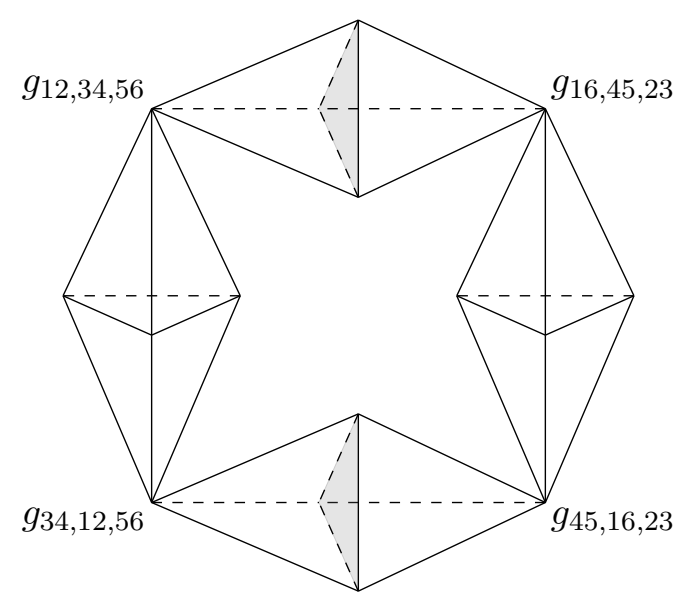

Figure 8. The arrangement of bipyramids inside $\operatorname{Tr}^{+}(3,6)$. The vertices represent the intersections of the rays $r_{i}$ with the unit sphere $S^{4}$. Two triangles are shaded to emphasize that they are actual 2 -faces of the polytope.

The regions of linearity of the tropical minors (6.6) define the fan for $\operatorname{Tr}^{+}(3,6)$ and its intersection with the unit sphere $S^{3}$ is a polytope with 16 vertices, 66 edges, 98 triangles and 48 three-dimensional facets. The tropical $\mathcal{X}$-coordinates are given by

$$
\begin{array}{ll}
\tilde{x}_{1}=w_{123}+w_{145}-w_{125}-w_{134}, & \tilde{x}_{3}=w_{124}+w_{156}-w_{126}-w_{145}, \\
\tilde{x}_{2}=w_{124}+w_{345}-w_{145}-w_{234}, & \tilde{x}_{4}=w_{134}+w_{125}+w_{456}-w_{124}-w_{156}-w_{345} .
\end{array}
$$

With the above relations (6.6) and (6.7) we can go back and forth between the representation of the 16 positive vertices in terms of the $e_{i j k}$ and in terms of a four-component representation which we can also obtain from cluster mutations as we now describe.

\subsection{Triangulating $\operatorname{Tr}^{+}(3,6)$ with clusters}

Unlike in $\operatorname{Gr}(2,5)$, the $\operatorname{Tr}^{+}(3,6)$ fan contains facets that are not simplicial. In particular, it contains 46 simplicial facets and two bipyramids defined by five vertices. This is a common feature of $k>2$ (tropical) Grassmannians.

To see this, first recall that $(k-1)(n-k+1)$ rays define a facet of the fan if an arbitrary positive linear combination of them solves the positive versions of inequalities derived from the Plücker relations. In particular $\operatorname{Tr}^{+}(3,6)$ has 2 such facets with five vertices that form bipyramids. These non-simplicial bipyramids are arranged inside the fan $\operatorname{Tr}^{+}(3,6)$ as sketched in figure 8 .

The fan $\operatorname{Tr}^{+}(3,6)$ is closely related to the dual of the $\mathrm{Gr}^{+}(3,6)$ associahedron in that the latter provides a natural triangulation of the former [13]. The vertices of the dual of the associahedron correspond to cluster $\mathcal{A}$-coordinates. Two vertices are connected by an edge when the corresponding pair of $\mathcal{A}$-coordinates appear together in a cluster, i.e. are cluster-adjacent in the sense of [11]. By definition, a pairwise connected quadruplet of vertices of the dual $\mathrm{Gr}^{+}(3,6)$ associahedron corresponds to a cluster, which in turn can be identified as a simplex triangulating $\operatorname{Tr}^{+}(3,6)$. 
We begin with the initial cluster

$$
\begin{gathered}
(124) \longrightarrow(125) \\
\downarrow \\
(134) \longrightarrow(145)
\end{gathered}
$$

and associate its unfrozen $\mathcal{A}$-coordinates with the unit vectors $\mathbf{e}_{1}, \ldots, \mathbf{e}_{4}$ in the tropical $\tilde{x}_{i}$ coordinates,

$$
\begin{array}{rlrl}
(124) & \leftrightarrow \mathbf{e}_{1}=(1,0,0,0), & (125) & \leftrightarrow \mathbf{e}_{2}=(0,1,0,0), \\
(134) & \leftrightarrow \mathbf{e}_{3}=(0,0,1,0), & (145) \leftrightarrow \mathbf{e}_{4}=(0,0,0,1) .
\end{array}
$$

Performing all possible mutations generates the full set of 16 ray vectors which arise from 50 distinct clusters.

Among the 16 rays we find the following five,

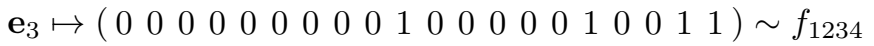

$$
\begin{aligned}
& -\mathbf{e}_{1} \mapsto\left(\begin{array}{lllllllllllllllllllll}
0 & 0 & 0 & 0 & 0 & -1 & -1 & -1 & -1 & -1 & 0 & -1 & -1 & -1 & -1 & -1 & -1 & -1 & -2 & -2
\end{array}\right) \sim f_{1256}
\end{aligned}
$$

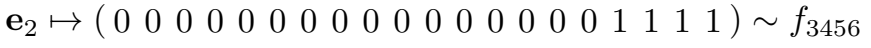

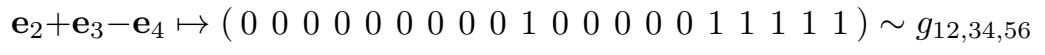

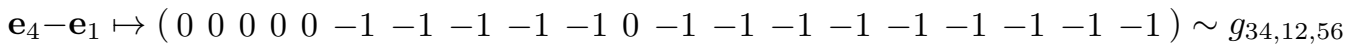

where we have also given their evaluations through the tropical minors (6.6) and the corresponding positive solutions given above. The fact that these five vertices form a single bipyramid rather than two tetrahedral facets can be seen from the linear relation,

$$
f_{1234}+f_{1256}+f_{3456}=g_{12,34,56}+g_{34,12,56} .
$$

Note that the cluster algebra provides a canonical way of determining a triangulation. In particular the bipyramid formed by the five rays described above is triangulated by two clusters whose vertices are given by $\left\{f_{1234}, f_{1256}, f_{3456}, g_{12,34,56}\right\}$ and $\left\{f_{1234}, f_{1256}, f_{3456}\right.$, $\left.g_{34,12,56}\right\}$.

Equipped with the cluster triangulation, we can express the scattering amplitude as a sum over clusters,

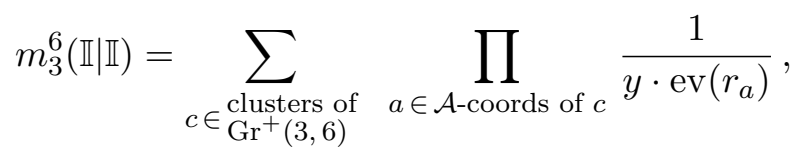

where as before $y=\left(s_{123}, \ldots, s_{456}\right)$ is the lexicographically ordered vector of Mandelstam invariants, $r_{a}$ is the representation of the $\mathcal{A}$-coordinate $a$ as a ray in $\tilde{x}$ coordinates and ev means the evaluation using the tropical minors in (6.6).

Using this identification, we can read off the two terms in the amplitude directly from the two clusters as ${ }^{2}$

$$
\frac{1}{t_{1234} t_{1256} t_{3456}}\left[\frac{1}{R_{12,34,56}}+\frac{1}{R_{34,12,56}}\right] .
$$

\footnotetext{
${ }^{2}$ Here we use the notation $t_{i j k l}=s_{i j k}+s_{i j l}+s_{i k l}+s_{j k l}$ and $R_{i j, k l, m n}=t_{i j k l}+s_{k l m}+s_{k l n}$.
} 
Note that using the identity between kinematic invariants

$$
R_{12,34,56}+R_{34,12,56}=t_{1234}+t_{3456}+t_{5612}
$$

we can write these two terms as

$$
(6.12)=\frac{1}{R_{12,34,56} R_{34,12,56}}\left[\frac{1}{t_{1256} t_{3456}}+\frac{1}{t_{1234} t_{3456}}+\frac{1}{t_{1234} t_{1256}}\right]
$$

which was noted in [1] to correspond to a different triangulation of the bipyramid. However the cluster algebra prefers a particular one of these triangulations.

\section{$7 \operatorname{Tr}(3,7):$ the amplitude from $E_{6}$ clusters}

In this section we explicitly demonstrate how the triangulation of the fan associated to the positive tropical Grassmannian $\operatorname{Tr}^{+}(3,7)$ can be worked out from the $\operatorname{Gr}(3,7)$ cluster algebra.

As in the previous section, one can either compute $F_{3,7}$ from the web $\mathrm{Web}_{3,7}$ or run the cluster-algebra machinery to obtain the generalised amplitude without even referring to $\operatorname{Tr}(3,7)$. Nevertheless let us first describe $\operatorname{Tr}^{+}(3,7)$ starting from $\operatorname{Tr}(3,7)$ and elaborate on a situation that is not encountered in Grassmannians of lower dimension.

The tropical Grassmannian $\operatorname{Tr}(3,7)$ has 721 rays which come in six types, ${ }^{3}$

$$
\begin{aligned}
& b_{1,1234567}=e_{123}, \\
& b_{2,1234567}=e_{123}+e_{124}+e_{134}+e_{234}, \\
& b_{3,1234567}=e_{123}+e_{124}+e_{125}+e_{126}+e_{127}, \\
& b_{4,1234567}=e_{123}+e_{124}+e_{125}+e_{126}+e_{127}+e_{134}+e_{234}, \\
& b_{5,1234567}=e_{123}+e_{124}+e_{125}+e_{126}+e_{127}+e_{134}+e_{156}+e_{234}+e_{256}, \\
& b_{6,1234567}=b_{3,1234567}+b_{3,3456712}+b_{3,6712345},
\end{aligned}
$$

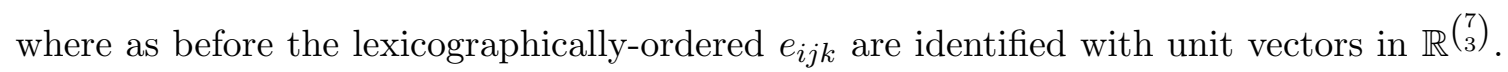
Other rays are obtained by the permutations of those that are written out above. For the types $b_{1}, \ldots, b_{5}$ and $b_{6}$, the permutations generate the six symmetry classes of respective sizes $35,35,21,210,315$ and 105 . These rays have also been tabulated in [18] with their explicit Plücker coordinates. Henceforth we will drop the labels in $b_{i, 1234567}$ and just write $b_{i}$ unless the order of the indices is not canonical.

To compute positive Grassmannian $\operatorname{Tr}^{+}(3,7)$, we select out of the 721 rays above those which solve the positive versions of tropicalised Plücker relations. One finds that 49 of them satisfy such relations. This seems incompatible with the fact that the cluster algebra has 42 distinct unfrozen $\mathcal{A}$-coordinates.

The resolution to this discrepancy is that seven positive rays of the type $b_{6}$ are linear combinations of three mutually-connected rays of type $b_{3}$, any positive linear combination

\footnotetext{
${ }^{3}$ This form was also given in [2].
} 
of which is a solution. In other words, $b_{6,1234567}$ is in the middle of a triangular 2-face of $\operatorname{Tr}^{+}(3,7)$ and is not necessary to define a cone of the fan.

As explained by Speyer and Williams [14], the $\operatorname{Tr}^{+}(3,7)$ fan has 693 facets. While 595 of these facets are simplicial, there are also 63 facets with 7 vertices, 28 with 8 vertices and 7 with 9 vertices. These non-simplicial facets are the $\operatorname{Gr}(3,7)$ analogues of the bipyramids of $\operatorname{Tr}^{+}(3,6)$.

We again resort to the relevant cluster algebra $E_{6}$ to obtain a triangulation on which we evaluate the amplitude. The $E_{6}$ cluster algebra has 833 clusters that give the vertices of the associahedron. These 833 clusters make up the simplexes of the triangulation each of which contain six vertices.

If we employ the duality between $\operatorname{Gr}(3,7)$ and $\operatorname{Gr}(4,7)$ and work in terms of the latter, we can relate the positive vertices above to the established notation for $\mathcal{A}$-coordinates in the literature on $\mathcal{N}=4$ amplitudes [10,11]. The different types of rays classified in (7.1a)(7.1f) nicely match the conventional cluster $\mathcal{A}$-coordinates:

$$
\begin{aligned}
a_{11} & \leftrightarrow b_{2,7123456} & a_{41} & \leftrightarrow b_{4,7156234} \\
a_{21} & \leftrightarrow b_{1,7123456} & a_{51} & \leftrightarrow b_{4,2345671} \\
a_{31} & \leftrightarrow b_{3,5671234} & a_{61} & \leftrightarrow b_{5,1234675}
\end{aligned}
$$

where the rest of the correspondence can be worked out by cyclic rotations of the second indices of the $a_{i j}$ and the arguments of the $b_{i}$. With this correspondence, we find that the $E_{6}$ initial cluster

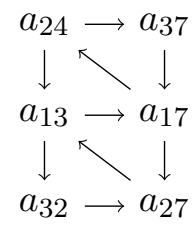

produces the following term in the amplitude

$$
\frac{1}{\left(y \cdot b_{1,1234567}\right)\left(y \cdot b_{3,1234567}\right)\left(y \cdot b_{2,1234567}\right)\left(y \cdot b_{2,4567123}\right)\left(y \cdot b_{3,6712345}\right)\left(y \cdot b_{1,5671234}\right)},
$$

with $y=\left(s_{123}, \ldots, s_{567}\right)$. We then mutate these rays according to (5.5) iteratively until we cover all 833 clusters of the $E_{6}$ polytope. Recovering the corresponding kinematic invariants using (7.2), we can construct the $\operatorname{Gr}(3,7)$ amplitude as the volume of the positive tropical Grassmannian. An expression for this amplitude is provided in the supplementary material Gr37amp.m.

\section{$8 \operatorname{Gr}(3,8):$ redundant triangulations}

In this section we will run the same construction in $\operatorname{Gr}(3,8)$ to provide a conjecture for the canonically-ordered part of the generalised biadjoint amplitude that one would obtain by solving the scattering equations for this Grassmannian. 
We start with the initial cluster of $\operatorname{Gr}(3,8)$

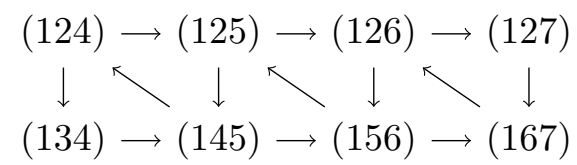

and identify its $\mathcal{A}$-coordinates with the rays (124) $\leftrightarrow \mathbf{e}_{\mathbf{1}},(134), \leftrightarrow \mathbf{e}_{\mathbf{2}},(125) \leftrightarrow \mathbf{e}_{\mathbf{3}}, \ldots$, $(167) \leftrightarrow \mathbf{e}_{8}$ in $\mathbb{R}^{8}$. Using the map explained in section 4.3 we recover the Plücker coordinates for the Speyer-Williams rays $\mathbf{e}_{1}, \ldots, \mathbf{e}_{8}$ and deduce that the initial cluster produces the following for the first term in the amplitude

$$
\begin{aligned}
& 1 /\left(\left(y \cdot b_{1,12345678}\right)\left(y \cdot b_{3,12345678}\right)\left(y \cdot b_{2,12345678}\right)\left(y \cdot b_{5,67548123}\right)\right. \\
& \left.\quad \times\left(y \cdot b_{5,34215678}\right)\left(y \cdot b_{2,56781234}\right)\left(y \cdot b_{3,78123456}\right)\left(y \cdot b_{1,67812345}\right)\right)
\end{aligned}
$$

where the $b$ vertices are given below in (8.4) and as before $y=\left(s_{123}, \ldots, s_{678}\right)$.

We then generate all 25,080 clusters using the mutation rules of [16] which we have adapted in equation (5.5). These clusters contain 128 distinct vectors in $\mathbb{R}^{8}$, identified with the $128 \mathcal{A}$-coordinates of $\operatorname{Gr}(3,8)$. As usual, the Plücker coordinates of these vectors provides us the factors in the denominator of every term in the amplitude. We provide all 25080 terms in the supplementary material Gr38amp.m.

Let us comment further on the correspondence between the $\operatorname{Tr}^{+}(3,8)$ fan and the $\operatorname{Gr}(3,8)$ cluster algebra. We find that, out of the 128 vectors generated by the cluster algebra, only 120 are rays of the corresponding fan. The extra 8 vectors have the form

$$
b_{\mathrm{e}}=b_{8,12345678}+b_{8,78564123}
$$

and cyclic rotations thereof. These too are positive vectors but being linear combinations of two genuine rays they lie on an edge of the fan. In other words, they separate only 7 regions of piecewise linearity for the tropical minors instead of 8 . This can be interpreted as the $\operatorname{Gr}(3,7)$ cluster algebra producing redundant triangulation of the fan which decomposes already simplicial facets into even smaller simplexes.

We can compare the Plücker coordinates of the vectors we obtain to the rays of another object called the Dressian $\operatorname{Dr}(3,8)$, studied in [19]. $\operatorname{Dr}(3,8)$ is a non-simplicial fan that consists of 15470 rays which split into 12 symmetry classes of size $(56,70,28,420,56,1260$, $420,560,1680,840,5040,5040)$. These define facets in groups of sizes ranging from 8 to 12. While all rays of $\operatorname{Dr}(k, n)$ are expected to be rays of $\operatorname{Gr}(k, n)$, the converse is not true. Indeed the Dressian $\operatorname{Dr}(3,8)$ does not capture the rays $b_{8}$ which give rise to "superfluous" triangulations.

The rays of $\operatorname{Dr}(3,8)$, positive and non-positive, are explicitly given as:

$$
\begin{aligned}
& b_{1}=e_{123}, \\
& b_{2}=e_{123}+e_{124}+e_{134}+e_{234}, \\
& b_{3}=e_{123}+e_{124}+e_{125}+e_{126}+e_{127}+e_{128}, \\
& b_{4}=e_{123}+e_{124}+e_{125}+e_{126}+e_{127}+e_{128}+e_{134}+e_{234},
\end{aligned}
$$




$$
\begin{aligned}
b_{5}= & e_{123}+e_{124}+e_{125}+e_{134}+e_{135}+e_{145}+e_{234}+e_{235}+e_{245}+e_{345}, \\
b_{6}= & e_{123}+e_{124}+e_{125}+e_{126}+e_{127}+e_{128}+e_{136}+e_{145}+e_{236}+e_{245}, \\
b_{7}= & e_{123}+e_{124}+e_{125}+e_{126}+e_{127}+e_{128}+e_{138}+e_{147}+e_{156}+e_{238} \\
& +e_{247}+e_{256}, \\
b_{8}= & e_{123}+e_{124}+e_{125}+e_{126}+e_{127}+e_{128}+e_{134}+e_{135}+e_{145}+e_{234} \\
& +e_{235}+e_{245}+e_{345}, \\
b_{9}= & e_{123}+e_{124}+e_{125}+e_{126}+e_{127}+e_{128}+e_{134}+e_{135}+e_{145}+e_{167} \\
& +e_{234}+e_{235}+e_{245}+e_{267}+e_{345}, \\
b_{10}= & e_{123}+e_{124}+e_{125}+e_{134}+e_{135}+e_{145}+e_{146}+e_{147}+e_{148}+e_{234} \\
& +e_{235}+e_{236}+e_{237}+e_{238}+e_{245}+e_{345}, \\
b_{11}= & e_{123}+e_{124}+e_{125}+e_{126}+e_{127}+e_{128}+e_{134}+e_{137}+e_{147}+e_{156} \\
& +e_{234}+e_{237}+e_{247}+e_{256}+e_{345}+e_{346}+e_{347}+e_{348}, \\
b_{12}= & e_{123}+e_{124}+e_{125}+e_{126}+e_{127}+e_{128}+e_{134}+e_{138}+e_{148}+e_{157} \\
& +e_{234}+e_{238}+e_{248}+e_{257}+e_{345}+e_{346}+e_{347}+e_{348}+e_{356}+e_{456} .
\end{aligned}
$$

Out of these, the 120 vectors defined by

$$
\begin{aligned}
& \left\{b_{1,12345678}, b_{2,12345678}, b_{3,12345678}, b_{4,12345678}, b_{4,23184567}, b_{5,23184567},\right. \\
& \quad b_{6,12378456}, b_{8,12345678}, b_{8,34128567}, b_{9,12345786}, b_{9,23178456}, b_{10,13482567} \\
& \left.\quad b_{11,34185627}, b_{11,12457836}, b_{12,12457683}\right\}
\end{aligned}
$$

and their cyclic copies lie in the positive region in the sense that they satisfy the positive version of the inequalities (3.5). These vectors are in one-to-one correspondence with the 120 non-redundant rays generated by the cluster algebra.

Note that the redundant vectors $b_{\mathrm{e}}$ that we encountered in $\operatorname{Gr}(3,8)$ are of different nature to the $b_{6}$ of $\operatorname{Gr}(3,7)$. While both types of vectors are not rays of the relevant fan, unlike the $b_{\mathrm{e}}$, the $b_{6}$ are not generated by the cluster algebra.

\section{Conclusions and outlook to $\operatorname{Gr}(4,8)$}

In this paper we have utilised cluster algebra technology to construct tree-level biadjoint amplitudes on $\operatorname{Gr}(3, n)$ for $n=6,7,8$. These amplitudes arise from scattering equations on the corresponding Grassmannians $[1,2]$ and the relevance of cluster algebras for these amplitudes arises from the interpretation of these amplitudes as volumes of certain geometric objects. In the cases we studied in this paper these objects are polyhedra in $(k-1)(n-k-1)-1$ dimensions, where $k=3$.

Cluster algebras provide a natural triangulation of the polyhedra whose volumes correspond to the scattering amplitudes. Therefore we were able to employ mutation rules to "bootstrap" the amplitude starting from a single term only. In particular we provided 
a prescription for the volume of the simplex that corresponds to the initial cluster and obtained the volumes of the remaining simplexes through consecutive cluster mutations.

Each of the cases we considered has new features that provide important lessons. In $n=6$ we saw that the clusters triangulate the bipyramids of $\operatorname{Tr}^{+}(3,6)$ into two simplexes. In $n=7$ we identified that positive rays that define cones of $\operatorname{Tr}^{+}(3,7)$ are not rays of the $\operatorname{Tr}^{+}(3,7)$ fan and are also not detected by the cluster algebra. When we studied the $n=8$ case, we found that the cluster algebra generates redundant triangulations of the $\operatorname{Tr}^{+}(3,8)$ fan.

Having studied the fans corresponding to various Grassmannians, a natural direction to take is to attempt to construct the fan for $\operatorname{Tr}^{+}(4,8)$, which corresponds to the positive part of $\operatorname{Gr}(4,8)$. The cluster algebra of the latter is expected to capture the rational symbol letters of 8-particle amplitudes in $\mathcal{N}=4$ but the fact that the $\operatorname{Gr}(4,8)$ cluster algebra is infinite has been a forbidding obstacle in utilising cluster algebras in the computations of these amplitudes.

We find that restricting the mutations to clusters that contain only rays obeying the full number of intersection conditions for $\operatorname{Tr}^{+}(4,8)$ closes on a finite number of 169,192 clusters. The corresponding $\mathcal{A}$-coordinates in these clusters provides us with a finite alphabet of 356 rational letters closed under cyclic rotations of the twistors. In particular, this alphabet contains the rational letters reported in [20]. It would be interesting to check if these letters are in correspondence with the faces of the polytope found by Arkani-Hamed, Lam and Spradlin, as reported in [21].

\section{Acknowledgments}

All authors are supported by ERC grant 648630 IQFT.

Open Access. This article is distributed under the terms of the Creative Commons Attribution License (CC-BY 4.0), which permits any use, distribution and reproduction in any medium, provided the original author(s) and source are credited.

\section{References}

[1] F. Cachazo, N. Early, A. Guevara and S. Mizera, Scattering Equations: From Projective Spaces to Tropical Grassmannians, JHEP 06 (2019) 039 [arXiv: 1903.08904] [InSPIRE].

[2] F. Cachazo and J.M. Rojas, Notes on Biadjoint Amplitudes, Trop $G(3,7)$ and $X(3,7)$ Scattering Equations, arXiv:1906.05979 [INSPIRE].

[3] F. Cachazo, S. He and E.Y. Yuan, Scattering equations and Kawai-Lewellen-Tye orthogonality, Phys. Rev. D 90 (2014) 065001 [arXiv:1306.6575] [InSPIRE].

[4] F. Cachazo, S. He and E.Y. Yuan, Scattering of Massless Particles in Arbitrary Dimensions, Phys. Rev. Lett. 113 (2014) 171601 [arXiv:1307.2199] [inSPIRE].

[5] F. Cachazo, S. He and E.Y. Yuan, Scattering of Massless Particles: Scalars, Gluons and Gravitons, JHEP 07 (2014) 033 [arXiv: 1309.0885] [INSPIRE].

[6] S. Fomin and A. Zelevinsky, Cluster algebras II: Finite type classification, Invent. Math. 154 (2003) 63 [math. RA/0208229]. 
[7] S. Fomin and A. Zelevinsky, Cluster algebras I: Foundations, J. Am. Math. Soc. 15 (2002) 497 [math.RT/0104151].

[8] J. Golden, A.B. Goncharov, M. Spradlin, C. Vergu and A. Volovich, Motivic Amplitudes and Cluster Coordinates, JHEP 01 (2014) 091 [arXiv: 1305.1617] [INSPIRE].

[9] N. Arkani-Hamed, Y. Bai, S. He and G. Yan, Scattering Forms and the Positive Geometry of Kinematics, Color and the Worldsheet, JHEP 05 (2018) 096 [arXiv:1711.09102] [INSPIRE].

[10] J.M. Drummond, G. Papathanasiou and M. Spradlin, A Symbol of Uniqueness: The Cluster Bootstrap for the 3-Loop MHV Heptagon, JHEP 03 (2015) 072 [arXiv: 1412.3763] [INSPIRE].

[11] J. Drummond, J. Foster and Ö. Gürdoğan, Cluster Adjacency Properties of Scattering Amplitudes in $N=4$ Supersymmetric Yang-Mills Theory, Phys. Rev. Lett. 120 (2018) 161601 [arXiv: 1710.10953] [INSPIRE].

[12] J. Drummond, J. Foster and Ö. Gürdoğan, Cluster adjacency beyond MHV, JHEP 03 (2019) 086 [arXiv: 1810.08149] [INSPIRE].

[13] S.B. Brodsky, C. Ceballos and J.-P. Labbé, Cluster Algebras of Type $D_{4}$, Tropical Planes, and the Positive Tropical Grassmannian, arXiv:1511.02699.

[14] D. Speyer and L.K. Williams, The tropical totally positive Grassmannian, math.C0/0312297.

[15] D. Speyer and B. Sturmfels, The tropical Grassmannian, Adv. Geom. 4 (2004) 389.

[16] S. Fomin and A. Zelevinsky, Cluster algebras IV: Coefficients, math.RA/0602259.

[17] N. Reading, A fan for every cluster algebra, talk given at the Combinatorics and beyond: the many facets of Sergey Fomin's mathematics, University of Michigan, Ann Arbor, Michigan, U.S.A., 8-11 November 2018.

[18] Planes in six dimensional tropical projective space, (2020) https://www.uni-math.gwdg.de/jensen/Research/G3_7/grassmann3_7.html.

[19] S. Herrmann, M. Joswig and D.E. Speyer, Dressians, tropical Grassmannians, and their rays, Forum Math. 26 (2012) 1853.

[20] I. Prlina, M. Spradlin, J. Stankowicz and S. Stanojevic, Boundaries of Amplituhedra and NMHV Symbol Alphabets at Two Loops, JHEP 04 (2018) 049 [arXiv: 1712.08049] [INSPIRE].

[21] A. Volovich, Cluster Algebras, Landau Singularities and Scattering Amplitudes, talk given at the Annual Theory Christmas Meeting, Durham, U.K., 17-19 December 2018. 